\title{
Quantum Cosmology: How to Interpret and Obtain Results
}

\author{
Nelson Pinto-Neto \\ Centro Brasileiro de Pesquisas Físicas, \\ Rua Dr. Xavier Sigaud 150, Urca \\ 22290-180, Rio de Janeiro, RJ, Brazil \\ E-mails: nelsonpn@lafex.cbpf.br
}

Received 7 January, 2000

\begin{abstract}
We argue that the Copenhagen interpretation of quantum mechanics cannot be applied to quantum cosmology. Among the alternative interpretations, we choose to apply the Bohm-de Broglie interpretation of quantum mechanics to canonical quantum cosmology. For minisuperspace models, we show that there is no problem of time in this interpretation, and that quantum effects can avoid the initial singularity, create inflation and isotropize the Universe. For the general case, it is shown that, irrespective of any regularization or choice of factor ordering of the Wheeler-DeWitt equation, the unique relevant quantum effect which does not break spacetime is the change of its signature from lorentzian to euclidean. The other quantum effects are either trivial or break the four-geometry of spacetime. A Bohm-de Broglie picture of a quantum geometrodynamics is constructed, which allows the investigation of these latter structures.
\end{abstract}

\section{Introduction}

Almost all physicists believe that quantum mechanics is a universal and fundamental theory, applicable to any physical system, from which classical physics can be recovered. The Universe is, of course, a valid physical system: there is a theory, Standard Cosmology, which is able to describe it in physical terms, and make predictions which can be confirmed or refuted by observations. In fact, the observations until now confirm the standard cosmological scenario. Hence, supposing the universality of quantum mechanics, the Universe itself must be described by quantum theory, from which we could recover Standard Cosmology. However, the Copenhagen interpretation of quantum mechanics $[1,2,3]^{1}$, which is the one taught in undergraduate courses and employed by the majority of physicists in all areas (specially the von Neumann's approach), cannot be used in a Quantum Theory of Cosmology. This is because it imposes the existence of a classical domain. In von Neumann's view, for instance, the necessity of a classical domain comes from the way it solves the measurement problem (see Ref. [4] for a good discussion). In an impulsive measurement of some observable, the wave function of the observed system plus macroscopic apparatus splits into many branches which almost do not overlap (in order to be a good measurement), each one containing the observed system in an eigenstate of the measured observable, and the pointer of the apparatus pointing to the respective eigenvalue. However, in the end of the measurement, we observe only one of these eigenvalues, and the measurement is robust in the sense that if we repeat it immediately after, we obtain the same result. So it seems that the wave function collapses, the other branches disappear. The Copenhagen interpretation assumes that this collapse is real. However, a real collapse cannot be described by the unitary Schrödinger evolution. Hence, the Copenhagen interpretation must assume that there is a fundamental process in a measurement which must occur outside the quantum world, in a classical domain. Of course, if we want to quantize the whole Universe, there is no place for a classical domain outside it, and the Copenhagen interpretation cannot be applied. Hence, if someone insists with the Copenhagen interpretation, she or he must assume that quantum theory is not universal, or at least try to improve it by means of further concepts. One possibility is by invoking the phenomenon of decoherence [5]. In fact, the interaction of the observed quantum system with its environment yields an effective diagonalization of the reduced density matrix, obtained by tracing out the irrelevant degrees of freedom. Decoherence can explain why the splitting of the wave

\footnotetext{
${ }^{1}$ Although these three authors have different views from quantum theory, the first emphasizing the indivisibility of quantum phenomena, the second with his notion of potentiality, and the third with the concept of quantum states, for all of them the existence of a classical domain is crucial. That is why we group their approaches under the same name "Copenhagen interpretation".
} 
function is given in terms of the pointer basis states, and why we do not see superpositions of macroscopic objects. In this way, classical properties emerge from quantum theory without the need of being assumed. In the framework of quantum gravity, it can also explain how a classical background geometry can emerge in a quantum universe [6]. In fact, it is the first quantity to become classical. However, decoherence is not yet a complete answer to the measurement problem $[7,8]$. It does not explain the apparent collapse after the measurement is completed, or why all but one of the diagonal elements of the density matrix become null when the measurement is finished. The theory is unable to give an account of the existence of facts, their uniqueness as opposed to the multiplicity of possible phenomena. Further developments are still in progress, like the consistent histories approach [9], which is however incomplete until now. The important role played by the observers in these descriptions is not yet explained [10], and still remains the problem on how to describe a quantum universe when the background geometry is not yet classical.

Nevertheless, there are some alternative solutions to this quantum cosmological dilemma which, together with decoherence, can solve the measurement problem maintaining the universality of quantum theory. One can say that the Schrödinger evolution is an approximation of a more fundamental non-linear theory which can accomplish the collapse $[11,12]$, or that the collapse is effective but not real, in the sense that the other branches disappear from the observer but do not disappear from existence. In this second category we can cite the Many-Worlds Interpretation [13] and the Bohm-de Broglie Interpretation [14, 15]. In the former, all the possibilities in the splitting are actually realized. In each branch there is an observer with the knowledge of the corresponding eigenvalue of this branch, but she or he is not aware of the other observers and the other possibilities because the branches do not interfere. In the latter, a point-particle in configuration space describing the observed system and apparatus is supposed to exist, independently on any observations. In the splitting, this point particle will enter into one of the branches (which one depends on the initial position of the point particle before the measurement, which is unknown), and the other branches will be empty. It can be shown [15] that the empty waves can neither interact with other particles, nor with the point particle containing the apparatus. Hence, no observer can be aware of the other branches which are empty. Again we have an effective but not real collapse (the empty waves continue to exist), but now with no multiplication of observers. Of course these interpretations can be used in quantum cosmology. Schrödinger evolution is always valid, and there is no need of a classical domain outside the observed system.

In this paper we review some results on the applica- tion of the Bohm-de Broglie interpretation to quantum cosmology $[16,17,18,19,20]$. In this approach, the fundamental object of quantum gravity, the geometry of 3-dimensional spacelike hypersurfaces, is supposed to exist independently on any observation or measurement, as well as its canonical momentum, the extrinsic curvature of the spacelike hypersurfaces. Its evolution, labeled by some time parameter, is dictated by a quantum evolution that is different from the classical one due to the presence of a quantum potential which appears naturally from the Wheeler-DeWitt equation. This interpretation has been applied to many minisuperspace models $[16,19,21,22,23,24]$, obtained by the imposition of homogeneity of the spacelike hypersurfaces. The classical limit, the singularity problem, the cosmological constant problem, and the time issue have been discussed. For instance, in some of these papers it was shown that in models involving scalar fields or radiation, which are nice representatives of the matter content of the early universe, the singularity can be clearly avoided by quantum effects. In the Bohm-de Broglie interpretation description, the quantum potential becomes important near the singularity, yielding a repulsive quantum force counteracting the gravitational field, avoiding the singularity and yielding inflation. The classical limit (given by the limit where the quantum potential becomes negligible with respect to the classical energy) for large scale factors are usually attainable, but for some scalar field models it depends on the quantum state and initial conditions. In fact it is possible to have small classical universes and large quantum ones [24]. About the time issue, it was shown that for any choice of the lapse function the quantum evolution of the homogeneous hypersurfaces yield the same four-geometry [19]. What remained to be studied is if this fact remains valid in the full theory, where we are not restricted to homogeneous spacelike hypersurfaces. The question is, given an initial hypersurface with consistent initial conditions, does the evolution of the initial three-geometry driven by the quantum bohmian dynamics yields the same four-geometry for any choice of the lapse and shift functions, and if it does, what kind of spacetime structure is formed? We know that this is true if the three-geometry is evolved by the dynamics of classical General Relativity (GR), yielding a non degenerate four geometry, but it can be false if the evolving dynamics is the quantum bohmian one. The idea was to put the quantum bohmian dynamics in hamiltonian form, and then use strong results presented in the literature exhibiting the most general form that a hamiltonian should have in order to form a non degenerate four-geometry from the evolution of threegeometries [25]. Our conclusion is that, in general, the quantum bohmian evolution of the three-geometries does not yield any non degenerate four-geometry at all [20]. Only for very special quantum states a relevant quantum non degenerate four-geometry can be 
obtained, and it must be euclidean. In the general case, the quantum bohmian evolution is consistent (still independent on the choice of the lapse and shift functions) but yielding a degenerate four-geometry, where special vector fields, the null eigenvectors of the four geometry, are present ${ }^{2}$. We arrived at these conclusions without assuming any regularization and factor ordering of the Wheeler-DeWitt equation. As we know, the WheelerDeWitt equation involves the application of the product of local operators on states at the same space point, which is ill defined [27]. Hence we need to regularize it in order to solve the factor ordering problem, and have a theory free of anomalies (for some proposals, see Refs $[28,29,30])$. Our conclusions are completely independent on these issues.

This paper is organized as follows: in the next section we review the Bohm-de Broglie interpretation of quantum mechanics for non-relativistic particles and quantum field theory in flat spacetime. In section III we apply the Bohm-de Broglie interpretation to canonical quantum gravity in the minisuperspace case. We show that there is no problem of time in this interpretation, and that quantum effects can avoid the initial singularity, create inflation, and isotropize the Universe. In section IV we treat the general case. We prove that the bohmian evolution of the 3-geometries, irrespective of any regularization and factor ordering of the Wheeler-DeWitt equation, can be obtained from a specific hamiltonian, which is of course different from the classical one. We then use this hamiltonian to obtain a picture of these new quantum structures. We end with conclusions and many perspectives for future work.

\section{The Bohm-de Broglie Inter- pretation}

In this section we will review the Bohm-de Broglie interpretation of quantum mechanics. We will first show how this interpretation works in the case of a single particle described by a Schrödinger equation, and then we will obtain, by analogy, the causal interpretation of quantum field theory in flat spacetime.

Let us begin with the Bohm-de Broglie interpretation of the Schrödinger equation describing a single particle. In the coordinate representation, for a nonrelativistic particle with Hamiltonian $H=p^{2} / 2 m+$ $V(x)$, the Schrödinger equation is

$$
i \hbar \frac{\partial \Psi(x, t)}{\partial t}=\left[-\frac{\hbar^{2}}{2 m} \nabla^{2}+V(x)\right] \Psi(x, t) .
$$

We can transform this differential equation over a complex field into a pair of coupled differential equations over real fields, by writing $\Psi=A \exp (i S / \hbar)$, where $A$ and $S$ are real functions, and substituting it into (1). We obtain the following equations.

$$
\begin{gathered}
\frac{\partial S}{\partial t}+\frac{(\nabla S)^{2}}{2 m}+V-\frac{\hbar^{2}}{2 m} \frac{\nabla^{2} A}{A}=0, \\
\frac{\partial A^{2}}{\partial t}+\nabla \cdot\left(A^{2} \frac{\nabla S}{m}\right)=0 .
\end{gathered}
$$

The usual probabilistic interpretation, i.e. the Copenhagen interpretation, understands equation (3) as a continuity equation for the probability density $A^{2}$ for finding the particle at position $x$ and time $t$. All physical information about the system is contained in $A^{2}$, and the total phase $S$ of the wave function is completely irrelevant. In this interpretation, nothing is said about $S$ and its evolution equation (2). Now suppose that the term $\frac{\hbar^{2}}{2 m} \frac{\nabla^{2} A}{A}$ is not present in Eqs. (2) and (3). Then we could interpret them as equations for an ensemble of classical particles under the influence of a classical potential $V$ through the Hamilton-Jacobi equation (2), whose probability density distribution in space $A^{2}(x, t)$ satisfies the continuity equation $(3)$, where $\nabla S(x, t) / m$ is the velocity field $v(x, t)$ of the ensemble of particles. When the term $\frac{\hbar^{2}}{2 m} \frac{\nabla^{2} A}{A}$ is present, we can still understand Eq. (2) as a Hamilton-Jacobi equation for an ensemble of particles. However, their trajectories are no more the classical ones, due to the presence of the quantum potential term in Eq. (2).

The Bohm-de Broglie interpretation of quantum mechanics is based on the two equations (2) and (3) in the way outlined above, not only on the last one as it is the Copenhagen interpretation. The starting idea is that the position $x$ and momentum $p$ are always well defined, with the particle's path being guided by a new field, the quantum field. The field $\Psi$ obeys Schrödinger equation (1), which can be written as the two real equations (2) and (3). Equation (2) is interpreted as a Hamilton-Jacobi type equation for the quantum particle subjected to an external potential, which is the classical potential plus the new quantum potential

$$
Q \equiv-\frac{\hbar^{2}}{2 m} \frac{\nabla^{2} A}{A} \text {. }
$$

Once the field $\Psi$, whose effect on the particle trajectory is through the quantum potential (4), is obtained from Schrödinger equation, we can also obtain the particle trajectory, $x(t)$, by integrating the differential equation $p=m \dot{x}=\nabla S(x, t)$, which is called the guidance relation (a dot means time derivative). Of course, from this differential equation, the non-classical trajectory $x(t)$ can only be known if the initial position of the particle is given. However, we do not know the initial

\footnotetext{
${ }^{2}$ For instance, the four geometry of Newtonian spacetime is degenerate [26], and its single null eigenvector is the normal of the absolute hypersurfaces of simultaneity, the time. As we know, it does not form a single spacetime structure because it is broken in absolute space plus absolute time.
} 
position of the particle because we do not know how to measure it without disturbances (it is the hidden variable of the theory). To agree with quantum mechanical experiments, we have to postulate that, for a statistical ensemble of particles in the same quantum field $\Psi$, the probability density distribution of initial positions $x_{0}$ is $P\left(x_{0}, t_{0}\right)=A^{2}\left(x_{0}, t=t_{0}\right)$. Equation (3) guarantees that $P(x, t)=A^{2}(x, t)$ for all times. In this way, the statistical predictions of quantum theory in the Bohm-de Broglie interpretation are the same as in the Copenhagen interpretation ${ }^{3}$.

It is interesting to note that the quantum potential depends only on the form of $\Psi$, not on its absolute value, as can be seen from equation (4). This fact brings home the non-local and contextual character of the quantum potential ${ }^{4}$. This is a necessary feature because Bell's inequalities together with Aspect's experiments show that, in general, a quantum theory must be either non-local or non-ontological. As the Bohmde Broglie interpretation is ontological, than it must be non-local, as it is. The non-local and contextual quantum potential causes the quantum effects. It has no parallel in classical physics.

The function $A$ plays a dual role in the Bohm-de Broglie interpretation: it gives the quantum potential and the probability density distribution of positions, but this last role is secondary. If in some model there is no notion of probability, we can still get information from the system using the guidance relations. In this case, $A^{2}$ does not need to be normalizable. The Bohmde Broglie interpretation is not, in essence, a probabilistic interpretation. It is straightforward to apply it to a single system.

The classical limit can be obtained in a very simple way. We only have to find the conditions for having $Q=0^{5}$. The question on why in a real measurement we see an effective collapse of the wave function is answered by noting that, in a measurement, the wave function splits in a superposition of non-overlapping branches. Hence the point particle (representing the particle being measured plus the macroscopic apparatus) will enter into one particular branch, which one depends on the initial conditions, and it will be influenced by the quantum potential related only to this branch, which is the only one that is not negligible in the region where the point particle actually is. The other empty branches continue to exist, but they neither influence on the point particle nor on any other particle [15]. There is an effective but not real collapse. The Schrödinger equation is always valid. There is no need to have a classical domain outside the quantum system to explain a measurement, neither is the existence of observers crucial because this interpretation is objective.

For quantum fields in flat spacetime, we can apply a similar reasoning. As an example, take the Schrödinger functional equation for a quantum scalar field:

$$
i \hbar \frac{\partial \Psi(\phi, t)}{\partial t}=\int d^{3} x\left\{\frac{1}{2}\left[-\hbar^{2} \frac{\delta^{2}}{\delta \phi^{2}}+(\nabla \phi)^{2}\right]+U(\phi)\right\} \Psi(\phi, t) .
$$

Writing again the wave functional as $\Psi=A \exp (i S / \hbar)$, we obtain:

$$
\begin{gathered}
\frac{\partial S}{\partial t}+\int d^{3} x\left\{\frac{1}{2}\left[\left(\frac{\delta S}{\delta \phi}\right)^{2}+(\nabla \phi)^{2}\right]+U(\phi)+Q(\phi)\right\}=0, \\
\frac{\partial A^{2}}{\partial t}+\int d^{3} x \frac{\delta}{\delta \phi}\left(A^{2} \frac{\delta S}{\delta \phi}\right)=0
\end{gathered}
$$

where $Q(\phi)=-\hbar^{2} \frac{1}{2 A} \frac{\delta^{2} A}{\delta \phi^{2}}$ is the corresponding (unregulated) quantum potential. The first equation is viewed as a modified Hamilton-Jacobi equation governing the evolution of some initial field configuration through time, which will be different from the classical

one due to the presence of the quantum potential. The guidance relation is now given by

$$
\Pi_{\phi}=\dot{\phi}=\frac{\delta S}{\delta \phi} .
$$

\footnotetext{
${ }^{3}$ It has been shown that under typical chaotic situations, and only within the Bohm-de Broglie interpretation, a probability distribution $P \neq A^{2}$ would rapidly approach the value $P=A^{2}[32,33]$. In this case, the probability postulate would be unnecessary, and we could have situations, in very short time intervals, where this modified Bohm-de Broglie interpretation would differ from the Copenhagen interpretation.

${ }^{4}$ The non-locality of $Q$ becomes evident when we generalize the causal interpretation to a many particles system.

${ }^{5}$ It should be very interesting to investigate the connection between this bohmian classical limit and the phenomenon of decoherence. To our knowledge, no work has ever been done on this issue, which may illuminate both the Bohm-de Broglie interpretation and the comprehension of decoherence.
} 
The second equation is the continuity equation for the probability density $A^{2}\left[\phi(x), t_{0}\right]$ of having the initial field configuration at time $t_{0}$ given by $\phi(x)$.

A detailed analysis of the Bohm-de Broglie interpretation of quantum field theory is given in Ref. [34] for the case of quantum electrodynamics.

\section{The Bohm-de Broglie In- terpretation of Minisuper- space Canonical Quantum Cosmology}

In this section, we summarize the rules of the Bohmde Broglie interpretation of quantum cosmology in the case of homogeneous minisuperspace models. When we are restricted to homogeneous models, the supermomentum constraint of GR is identically zero, and the shift function can be set to zero without loosing any of the Einstein's equations. The hamiltonian is reduced to general minisuperspace form:

$$
H_{G R}=N(t) \mathcal{H}\left(p^{\alpha}(t), q_{\alpha}(t)\right),
$$

where $p^{\alpha}(t)$ and $q_{\alpha}(t)$ represent the homogeneous degrees of freedom coming from $\Pi^{i j}(x, t)$ and $h_{i j}(x, t)$. The minisuperspace Wheeler-De Witt equation is:

$$
\mathcal{H}\left(\hat{p}^{\alpha}(t), \hat{q}_{\alpha}(t)\right) \Psi(q)=0 .
$$

Writing $\Psi=R \exp (i S / \hbar)$, and substituting it into (10), we obtain the following equation:

$$
\frac{1}{2} f_{\alpha \beta}\left(q_{\mu}\right) \frac{\partial S}{\partial q_{\alpha}} \frac{\partial S}{\partial q_{\beta}}+U\left(q_{\mu}\right)+Q\left(q_{\mu}\right)=0
$$

where

$$
Q\left(q_{\mu}\right)=-\frac{1}{R} f_{\alpha \beta} \frac{\partial^{2} R}{\partial q_{\alpha} \partial q_{\beta}},
$$

and $f_{\alpha \beta}\left(q_{\mu}\right)$ and $U\left(q_{\mu}\right)$ are the minisuperspace particularizations of the DeWitt metric $G_{i j k l}[36]$ and of the scalar curvature density $-h^{1 / 2} R^{(3)}\left(h_{i j}\right)$ of the spacelike hypersurfaces, respectively. The causal interpretation applied to quantum cosmology states that the trajectories $q_{\alpha}(t)$ are real, independently of any observations. Eq. (11) is the Hamilton-Jacobi equation for them, which is the classical one amended with a quantum potential term (12), responsible for the quantum effects. This suggests to define:

$$
p^{\alpha}=\frac{\partial S}{\partial q_{\alpha}}
$$

where the momenta are related to the velocities in the usual way:

$$
p^{\alpha}=f^{\alpha \beta} \frac{1}{N} \frac{\partial q_{\beta}}{\partial t} .
$$

To obtain the quantum trajectories we have to solve the following system of first order differential equations:

$$
\frac{\partial S\left(q_{\alpha}\right)}{\partial q_{\alpha}}=f^{\alpha \beta} \frac{1}{N} \frac{\partial q_{\beta}}{\partial t} .
$$

Eqs. (15) are invariant under time reparametrization. Hence, even at the quantum level, different choices of $N(t)$ yield the same spacetime geometry for a given non-classical solution $q_{\alpha}(t)$. There is no problem of time in the causal interpretation of minisuperspace quantum cosmology.

Let us now apply these rules, as examples, to minisuperspace models with a free massless scalar field. Take the lagrangian:

$$
L=\sqrt{-g} e^{-\phi}\left(R-w \phi_{; \rho} \phi^{; \rho}\right) .
$$

For $w=-1$ we have effective string theory without the Kalb-Rammond field. For $w=-3 / 2$ we have a conformally coupled scalar field. Performing the conformal transformation $g_{\mu \nu}=e^{\phi} \bar{g}_{\mu \nu}$ we obtain the following lagrangian:

$$
L=\sqrt{-g}\left[R-\left(\omega+\frac{3}{2}\right) \phi_{; \rho} \phi^{; \rho}\right] \quad,
$$

where the bars have been omitted. We will define $C_{w} \equiv\left(\omega+\frac{3}{2}\right)$.

\section{III.1. The isotropic case}

We consider now the Robertson-Walker metric

$$
d s^{2}=-N^{2} \mathrm{~d} t^{2}+\frac{a(t)^{2}}{1+\frac{\epsilon}{4} r^{2}}\left[\mathrm{~d} r^{2}+r^{2}\left(\mathrm{~d} \theta^{2}+\sin ^{2}(\theta) \mathrm{d} \varphi^{2}\right)\right]
$$

where the spatial curvature $\epsilon$ takes the values $0,1,-1$. Inserting this in the lagrangian (17), and using the units where $\hbar=c=1$, we obtain the following action:

$$
S=\frac{3 V}{4 \pi l_{p}^{2}} \int \frac{N a^{3}}{2}\left(\frac{-\dot{a}^{2}}{N^{2} a^{2}}+C_{w} \frac{\dot{\phi}^{2}}{6 N^{2}}+\frac{\epsilon}{a^{2}}\right) \mathrm{d} t,
$$

where $V$ is the total volume divided by $a^{3}$ of the spacelike hypersurfaces, which are supposed to be closed, and $l_{p}$ is the Planck length. $V$ depends on the value of $\epsilon$ and on the topology of the hypersurfaces. For $\epsilon=0$ it can be as large as we want because their fundamental polyhedra can have arbitrary size. In the case of $\epsilon=1$ and topology $S^{3}, V=2 \pi^{2}$. Defining $\beta^{2}=\frac{4 \pi l_{p}^{2}}{3 V}$, the hamiltonian turns out to be:

$$
H=N\left(-\beta^{2} \frac{p_{a}^{2}}{2 a}+3 \beta^{2} \frac{p_{\phi}^{2}}{C_{w} a^{3}}-\epsilon \frac{a}{2 \beta^{2}}\right) .
$$

where 


$$
\begin{aligned}
& p_{a}=-\frac{a \dot{a}}{\beta^{2} N}, \\
& p_{\phi}=C_{w} \frac{a^{3} \dot{\phi}}{6 \beta^{2} N}
\end{aligned}
$$

Usually the scale factor has dimensions of length because we use angular coordinates in closed spaces. Hence we will define a dimensionless scale factor $\tilde{a} \equiv$ $a / \beta$. In that case the hamiltonian becomes, omitting the tilde:

$$
H=\frac{N}{2 \beta}\left(-\frac{p_{a}^{2}}{a}+6 \frac{p_{\phi}^{2}}{C_{w} a^{3}}-\epsilon a\right) .
$$

As $\beta$ appears as an overall multiplicative constant in the hamiltonian, we can set it equal to one without any loss of generality, keeping in mind that the scale factor which appears in the metric is $\beta a$, not $a$. We can further simplify the hamiltonian by defining $\alpha \equiv \ln (a)$ obtaining

$$
H=\frac{N}{2 \exp (3 \alpha)}\left[-p_{\alpha}^{2}+\frac{6}{C_{w}} p_{\phi}^{2}-\epsilon \exp (4 \alpha)\right] .
$$

where

$$
\begin{aligned}
& p_{\alpha}=-\frac{e^{3 \alpha} \dot{\alpha}}{N}, \\
& p_{\phi}=C_{w} \frac{e^{3 \alpha} \dot{\phi}}{6 N} .
\end{aligned}
$$

The momentum $p_{\phi}$ is a constant of motion which we will call $\bar{k}$. We will restrict ourselves to the physically interesting case, due to observations, of $\epsilon=0$ and $C_{w}>0$.

The classical solutions in the gauge $N=1$ are,

$$
\phi= \pm \sqrt{\frac{6}{C_{w}}} \alpha+c_{1}
$$

where $c_{1}$ is an integration constant. In term of cosmic time they are:

$$
\begin{aligned}
a & =e^{\alpha}=3 \sqrt{\frac{6}{C_{w}}} \bar{k} t^{1 / 3}, \\
\phi & =\sqrt{\frac{2}{3 C_{w}}} \ln (t)+c_{2} .
\end{aligned}
$$

The solutions contract or expand forever from a singularity, depending on the sign of $\bar{k}$, without any inflationary epoch.

Let us now quantize the model. With a particular choice of factor ordering, we obtain the following Wheeler-DeWitt equation

$$
\Psi_{\alpha \alpha}-\frac{6}{C_{w}} \Psi_{\phi \phi}-\epsilon e^{4 \alpha} \Psi=0
$$

Employing the separation of variables method, we obtain the general solution

$$
\Psi(\alpha, \phi)=\int F(k) A_{k}(\alpha) B_{k}(\phi) d k
$$

where $k$ is a separation constant, and

$$
B_{k}(\phi)=b_{1} \exp \left(i \sqrt{\frac{C_{w}}{6}} k \phi\right)+b_{2} \exp \left(-i \sqrt{\frac{C_{w}}{6}} k \phi\right)
$$

and

$$
A_{k}(\alpha)=a_{1} \exp (i k \alpha)+a_{2} \exp (-i k \alpha),
$$

We will now make gaussian superpositions of these solutions and interpret the results using the causal interpretation of quantum mechanics. The function $F(k)$ is:

$$
F(k)=\exp \left[-\frac{(k-d)^{2}}{\sigma^{2}}\right] .
$$

We take the wave function:

$$
\Psi(\alpha, \phi)=\int F(k)\left[A_{k}(\alpha) B_{k}(\phi)+A_{-k}(\alpha) B_{k}(\phi) d k\right]
$$

with $a_{2}=b_{2}=0$.

Performing the integration in $k$ we obtain for $\Psi$ (we will define $\bar{\phi} \equiv \sqrt{\frac{C_{w}}{6}} \phi$ and omit the bars from now on)

$$
\Psi=\sigma \sqrt{\pi}\left\{\exp \left[-\frac{(\alpha+\phi)^{2} \sigma^{2}}{4}\right] \exp [i d(\alpha+\phi)]+\exp \left[-\frac{(\alpha-\phi)^{2} \sigma^{2}}{4}\right] \exp [-i d(\alpha-\phi)]\right\}
$$


In order to obtain the Bohmian trajectories, we have to calculate the phase $S$ of the above wave function and substitute it into the guidance formula

$$
\begin{aligned}
& p_{\alpha}=S_{\alpha}, \\
& p_{\phi}=S_{\phi},
\end{aligned}
$$

where

$$
\begin{aligned}
& p_{\alpha}=-\frac{e^{3 \alpha} \dot{\alpha}}{N}, \\
& p_{\phi}=\frac{e^{3 \alpha} \dot{\phi}}{N} .
\end{aligned}
$$

We will work in the gauge $N=1$. These equations constitute a planar system which can be easily studied:

$$
\begin{aligned}
\dot{\alpha} & =\frac{\left[\phi \sigma^{2} \sin (2 d \alpha)+2 d \sinh \left(\sigma^{2} \alpha \phi\right)\right]}{\exp (3 \alpha)\left\{2\left[\cos (2 d \alpha)+\cosh \left(\sigma^{2} \alpha \phi\right)\right]\right\}} \\
\dot{\phi} & =\frac{\left[\alpha \sigma^{2} \sin (2 d \alpha)-2 d \cos (2 d \alpha)-2 \cosh \left(\sigma^{2} \alpha \phi\right)\right]}{\exp (3 \alpha)\left\{2\left[\cos (2 d \alpha)+\cosh \left(\sigma^{2} \alpha \phi\right)\right]\right\}}
\end{aligned}
$$

The line $\alpha=0$ divides configuration space in two symmetric regions. The line $\phi=0$ contains all singular points of this system, which are nodes and centers. The nodes appear when the denominator of the above equations, which is proportional to the norm of the wave function, is zero. No trajectory can pass through these points. They happen when $\phi=0$ and $\cos (d \alpha)=0$, or $\alpha=(2 n+1) \pi / 2 d, n$ an integer, with separation $\pi / d$. The center points appear when the numerators are zero. They are given by $\phi=0$ and $\alpha=2 d[\operatorname{cotan}(d \alpha)] / \sigma^{2}$. They are intercalated with the node points. As $|\alpha| \rightarrow \infty$ these points tend to $n \pi / d$, and their separations cannot exceed $\pi / d$. As one can see from the above system, the classical solutions $\left(a(t) \propto t^{1 / 3}\right)$ are recovered when $|\alpha| \rightarrow \infty$ or $|\phi| \rightarrow \infty$, the other being different from zero.

There are plenty of different possibilities of evolution, depending on the initial conditions. Near the center points we can have oscillating universes without singularities and with amplitude of oscillation of order 1 . For negative values of $\alpha$, the universe arise classically from a singularity but quantum effects become important forcing it to recollapse to another singularity, recovering classical behaviour near it. For positive values of $\alpha$, the universe contracts classically but when $\alpha$ is small enough quantum effects become important creating an inflationary phase which avoids the singularity. The universe contracts to a minimum size and after reaching this point it expands forever, recovering the classical limit when $\alpha$ becomes sufficiently large. We can see that for $\alpha$ negative we have classical limit for small scale factor while for $\alpha$ positive we have classical limit for big scale factor.

\section{III.2. The anisotropic case}

To exemplify the quantum isotropization of the Universe, let us take now, instead of the FriedmanRobertson-Walker of Eq. (18), the homogeneous and anisotropic Bianchi I line element

$$
\begin{aligned}
d s^{2}= & -N^{2}(t) d t^{2}+\exp \left[2 \beta_{0}(t)+2 \beta_{+}(t)+2 \sqrt{3} \beta_{-}(t)\right] d x^{2}+ \\
& \exp \left[2 \beta_{0}(t)+2 \beta_{+}(t)-2 \sqrt{3} \beta_{-}(t)\right] d y^{2}+ \\
& \exp \left[2 \beta_{0}(t)-4 \beta_{+}(t)\right] d z^{2} .
\end{aligned}
$$

This line element will be isotropic if and only if $\beta_{+}(t)$ and $\beta_{-}(t)$ are constants. Inserting Eq. (43) into the lagrangian (17), supposing that the scalar field $\phi$ depends only on time, discarding surface terms, and performing a Legendre transformation, we obtain the following minisuperspace classical hamiltonian

$$
H=\frac{N}{24 \exp \left(3 \beta_{0}\right)}\left(-p_{0}^{2}+p_{+}^{2}+p_{-}^{2}+p_{\phi}^{2}\right)
$$

where $\left(p_{0}, p_{+}, p_{-}, p_{\phi}\right)$ are canonically conjugate to $\left(\beta_{0}, \beta_{+}, \beta_{-}, \phi\right)$, respectively, and we made the trivial redefinition $\phi \rightarrow \sqrt{C_{w} / 6} \phi$.

We can write this hamiltonian in a compact form by defining $y^{\mu}=\left(\beta_{0}, \beta_{+}, \beta_{-}, \phi\right)$ and their canonical momenta $p_{\mu}=\left(p_{0}, p_{+}, p_{-}, p_{\phi}\right)$, obtaining

$$
H=\frac{N}{24 \exp \left(3 y^{0}\right)} \eta^{\mu \nu} p_{\mu} p_{\nu},
$$

where $\eta^{\mu \nu}$ is the Minkowski metric with signature $(-+++)$. The equations of motion are the constraint equation obtained by varying the hamiltonian with re- 
spect to the lapse function $N$

$$
\mathcal{H} \equiv \eta^{\mu \nu} p_{\mu} p_{\nu}=0,
$$

and the Hamilton's equations

$$
\begin{aligned}
\dot{y}^{\mu}=\frac{\partial \mathcal{H}}{\partial p_{\mu}} & =\frac{N}{12 \exp \left(3 y_{0}\right)} \eta^{\mu \nu} p_{\nu}, \\
\dot{p}_{\mu} & =-\frac{\partial \mathcal{H}}{\partial y^{\mu}}=0 .
\end{aligned}
$$

The solution to these equations in the gauge $N=$ $12 \exp \left(3 y_{0}\right)$ is

$$
y^{\mu}=\eta^{\mu \nu} p_{\nu} t+C^{\mu}
$$

where the momenta $p_{\nu}$ are constants due to the equations of motion and the $C^{\mu}$ are integration constants. We can see that the only way to obtain isotropy in these solutions is by making $p_{1}=p_{+}=0$ and $p_{2}=$ $p_{-}=0$, which yield solutions that are always isotropic, the usual Friedman-Robertson-Walker (FRW) solutions with a scalar field. Hence, there is no anisotropic solution in this model which can classically become isotropic during the course of its evolution. Once anisotropic, always anisotropic. If we suppress the $\phi$ degree of freedom, the unique isotropic solution is flat spacetime because in this case the constraint (46) enforces $p_{0}=0$.

To discuss the appearance of singularities, we need the Weyl square tensor $W^{2} \equiv W^{\alpha \beta \mu \nu} W_{\alpha \beta \mu \nu}$. It reads

$$
W^{2}=\frac{1}{432} e^{-12 \beta_{0}}\left(2 p_{0} p_{+}^{3}-6 p_{0} p_{-}^{2} p_{+}+p_{-}^{4}+2 p_{+}^{2} p_{-}^{2}+p_{+}^{4}+p_{0}^{2} p_{+}^{2}+p_{0}^{2} p_{-}^{2}\right)
$$

Hence, the Weyl square tensor is proportional to $\exp \left(-12 \beta_{0}\right)$ because the $p$ 's are constants (see Eq. (48)) and the singularity is at $t=-\infty$. The classical singularity can be avoided only if we set $p_{0}=0$. But then, due to equation (46), we would also have $p_{i}=0$, which corresponds to the trivial case of flat spacetime. Hence, the unique classical solution which is non-singular is the trivial flat spacetime solution.

The Dirac quantization procedure yields the Wheeler-DeWitt equation, which in the present case reads

$$
\eta^{\mu \nu} \frac{\partial^{2}}{\partial y^{\mu} y^{\nu}} \Psi\left(y^{\mu}\right)=0
$$

Let us now investigate spherical-wave solutions of Eq. (51). They read

$$
\Psi_{3}=\frac{1}{y}\left[f\left(y^{0}+y\right)+g\left(y^{0}-y\right)\right]
$$

where $y \equiv \sqrt{\sum_{i=1}^{3}\left(y^{i}\right)^{2}}$.

The guidance relations in the gauge $N=$ $12 \exp \left(3 y_{0}\right)$ are (see Eqs. (47)) read

$$
\begin{gathered}
p_{0}=\partial_{0} S=\operatorname{Im}\left(\frac{\partial_{0} \Psi_{3}}{\Psi_{3}}\right)=-\dot{y}^{0}, \\
p_{i}=\partial_{i} S=\operatorname{Im}\left(\frac{\partial_{i} \Psi_{3}}{\Psi_{3}}\right)=\dot{y}^{i},
\end{gathered}
$$

where $S$ is the phase of the wave function. In terms of $f$ and $g$ the above equations read

$$
\dot{y}^{0}=-\operatorname{Im}\left(\frac{f^{\prime}\left(y^{0}+y\right)+g^{\prime}\left(y^{0}-y\right)}{f\left(y^{0}+y\right)+g\left(y^{0}-y\right)}\right),
$$

$$
\dot{y}^{i}=\frac{y^{i}}{y} \operatorname{Im}\left(\frac{f^{\prime}\left(y^{0}+y\right)-g^{\prime}\left(y^{0}-y\right)}{f\left(y^{0}+y\right)+g\left(y^{0}-y\right)}\right),
$$

where the prime means derivative with respect to the argument of the functions $f$ and $g$, and $\operatorname{Im}(z)$ is the imaginary part of the complex number $z$.

From Eq. (56) we obtain that

$$
\frac{d y^{i}}{d y^{j}}=\frac{y^{i}}{y^{j}},
$$

which implies that $y^{i}(t)=c_{j}^{i} y^{j}(t)$, with no sum in $j$, where the $c_{j}^{i}$ are real constants and $c_{1}^{1}=c_{2}^{2}=c_{3}^{3}=$ 1. Hence, apart some positive multiplicative constant, knowing about one of the $y^{i}$ means knowing about all $y^{i}$. Consequently, we can reduce the four equations (55) and (56) to a planar system by writing $y=C\left|y^{3}\right|$, with $C>1$, and working only with $y^{0}$ and $y^{3}$, say. The planar system now reads

$$
\begin{gathered}
\dot{y}^{0}=-\operatorname{Im}\left(\frac{f^{\prime}\left(y^{0}+C\left|y^{3}\right|\right)+g^{\prime}\left(y^{0}-C\left|y^{3}\right|\right)}{f\left(y^{0}+C\left|y^{3}\right|\right)+g\left(y^{0}-C\left|y^{3}\right|\right)}\right), \\
\dot{y}^{3}=\frac{\operatorname{sign}\left(y^{3}\right)}{C} \operatorname{Im}\left(\frac{f^{\prime}\left(y^{0}+C\left|y^{3}\right|\right)-g^{\prime}\left(y^{0}-C\left|y^{3}\right|\right)}{f\left(y^{0}+C\left|y^{3}\right|\right)+g\left(y^{0}-C\left|y^{3}\right|\right)}\right) .
\end{gathered}
$$

Note that if $f=g, y^{3}$ stabilizes at $y^{3}=0$ because $\dot{y}^{3}$ as well as all other time derivatives of $y^{3}$ are zero at this line. As $y^{i}(t)=c_{j}^{i} y^{j}(t)$, all $y^{i}(t)$ become zero, and the cosmological model isotropizes forever once $y^{3}$ reaches this line. Of course one can find solutions where $y^{3}$ never reaches this line, but in this case there must be some region where $\dot{y}^{3}=0$, which implies $\dot{y}^{i}=0$, and this is an isotropic region. Consequently, quantum anisotropic cosmological models with $f=g$ always 
have an isotropic phase, which can become permanent in many cases.

\section{The Bohm-de Broglie In- terpretation of Superspace Canonical Quantum Cos- mology}

In this section, we will quantize General Relativity Theory (GR) without making any simplifications or cutting of degrees of freedom. The matter content is a minimally coupled scalar field with arbitrary potential. All subsequent results remain essentially the same for any matter field which couples uniquely with the metric, not with their derivatives.

The classical hamiltonian of full GR with a scalar field is given by:

$$
H=\int d^{3} x\left(N \mathcal{H}+N^{j} \mathcal{H}_{j}\right)
$$

where

$$
\begin{aligned}
\mathcal{H}= & \kappa G_{i j k l} \Pi^{i j} \Pi^{k l}+\frac{1}{2} h^{-1 / 2} \Pi_{\phi}^{2}+ \\
& +h^{1 / 2}\left[-\kappa^{-1}\left(R^{(3)}-2 \Lambda\right)+\frac{1}{2} h^{i j} \partial_{i} \phi \partial_{j} \phi+U(\phi)\right] \\
\mathcal{H}_{j}= & -2 D_{i} \Pi_{j}^{i}+\Pi_{\phi} \partial_{j} \phi .
\end{aligned}
$$

In these equations, $h_{i j}$ is the metric of closed 3dimensional spacelike hypersurfaces, and $\Pi^{i j}$ is its canonical momentum given by

$\Pi^{i j}=-h^{1 / 2}\left(K^{i j}-h^{i j} K\right)=G^{i j k l}\left(\dot{h}_{k l}-D_{k} N_{l}-D_{l} N_{k}\right)$,

where

$$
K_{i j}=-\frac{1}{2 N}\left(\dot{h}_{i j}-D_{i} N_{j}-D_{j} N_{i}\right),
$$

is the extrinsic curvature of the hypersurfaces (indices are raised and lowered by the 3-metric $h_{i j}$ and its inverse $\left.h^{i j}\right)$. The canonical momentum of the scalar field is now

$$
\Pi_{\phi}=\frac{h^{1 / 2}}{N}\left(\dot{\phi}-N^{i} \partial_{i} \phi\right) .
$$

The quantity $R^{(3)}$ is the intrinsic curvature of the hypersurfaces and $h$ is the determinant of $h_{i j}$. The lapse function $N$ and the shift function $N_{j}$ are the Lagrange multipliers of the super-hamiltonian constraint $\mathcal{H} \approx 0$ and the super-momentum constraint $\mathcal{H}^{j} \approx 0$, respectively. They are present due to the invariance of GR under spacetime coordinate transformations. The quantities $G_{i j k l}$ and its inverse $G^{i j k l}\left(G_{i j k l} G^{i j a b}=\delta_{k l}^{a b}\right)$ are given by

$$
\begin{aligned}
& G^{i j k l}=\frac{1}{2} h^{1 / 2}\left(h^{i k} h^{j l}+h^{i l} h^{j k}-2 h^{i j} h^{k l}\right), \\
& G_{i j k l}=\frac{1}{2} h^{-1 / 2}\left(h_{i k} h_{j l}+h_{i l} h_{j k}-h_{i j} h_{k l}\right),
\end{aligned}
$$

which is called the DeWitt metric. The quantity $D_{i}$ is the $i$-component of the covariant derivative operator on the hypersurface, and $\kappa=16 \pi G / c^{4}$.

The classical 4-metric

$$
d s^{2}=-\left(N^{2}-N^{i} N_{i}\right) d t^{2}+2 N_{i} d x^{i} d t+h_{i j} d x^{i} d x^{j}
$$

and the scalar field which are solutions of the Einstein's equations can be obtained from the Hamilton's equations of motion

$$
\begin{aligned}
\dot{h}_{i j} & =\left\{h_{i j}, H\right\}, \\
\dot{\Pi}^{i j} & =\left\{\Pi^{i j}, H\right\}, \\
\dot{\phi} & =\{\phi, H\}, \\
\dot{\Pi}_{\phi} & =\left\{\Pi_{\phi}, H\right\},
\end{aligned}
$$

for some choice of $N$ and $N^{i}$, and if we impose initial conditions compatible with the constraints

$$
\begin{aligned}
\mathcal{H} & \approx 0, \\
\mathcal{H}_{i} & \approx 0 .
\end{aligned}
$$

It is a feature of the hamiltonian of GR that the 4metrics (68) constructed in this way, with the same initial conditions, describe the same four-geometry for any choice of $N$ and $N^{i}$. The algebra of the constraints close in the following form (we follow the notation of Ref. [25]): 


$$
\begin{aligned}
\left\{\mathcal{H}(x), \mathcal{H}\left(x^{\prime}\right)\right\} & =\mathcal{H}^{i}(x) \partial_{i} \delta^{3}\left(x, x^{\prime}\right)-\mathcal{H}^{i}\left(x^{\prime}\right) \partial_{i} \delta^{3}\left(x^{\prime}, x\right) \\
\left\{\mathcal{H}_{i}(x), \mathcal{H}\left(x^{\prime}\right)\right\} & =\mathcal{H}(x) \partial_{i} \delta^{3}\left(x, x^{\prime}\right) \\
\left\{\mathcal{H}_{i}(x), \mathcal{H}_{j}\left(x^{\prime}\right)\right\} & =\mathcal{H}_{i}(x) \partial_{j} \delta^{3}\left(x, x^{\prime}\right)+\mathcal{H}_{j}\left(x^{\prime}\right) \partial_{i} \delta^{3}\left(x, x^{\prime}\right)
\end{aligned}
$$

To quantize this constrained system, we follow the Dirac quantization procedure. The constraints become conditions imposed on the possible states of the quantum system, yielding the following quantum equations:

$$
\begin{aligned}
\hat{\mathcal{H}}_{i} \mid \Psi> & =0 \\
\hat{\mathcal{H}} \mid \Psi> & =0
\end{aligned}
$$

In the metric and field representation, the first equation is

$$
-2 h_{l i} D_{j} \frac{\delta \Psi\left(h_{i j}, \phi\right)}{\delta h_{l j}}+\frac{\delta \Psi\left(h_{i j}, \phi\right)}{\delta \phi} \partial_{i} \phi=0,
$$

which implies that the wave functional $\Psi$ is an invariant under space coordinate transformations.

The second equation is the Wheeler-DeWitt equation $[35,36]$. Writing it unregulated in the coordinate representation we get

$$
\left\{-\hbar^{2}\left[\kappa G_{i j k l} \frac{\delta}{\delta h_{i j}} \frac{\delta}{\delta h_{k l}}+\frac{1}{2} h^{-1 / 2} \frac{\delta^{2}}{\delta \phi^{2}}\right]+V\right\} \Psi\left(h_{i j}, \phi\right)=0,
$$

where $V$ is the classical potential given by

$$
V=h^{1 / 2}\left[-\kappa^{-1}\left(R^{(3)}-2 \Lambda\right)+\frac{1}{2} h^{i j} \partial_{i} \phi \partial_{j} \phi+U(\phi)\right] .
$$

This equation involves products of local operators at the same space point, hence it must be regularized. After doing this, one should find a factor ordering which makes the theory free of anomalies, in the sense that the commutator of the operator version of the constraints close in the same way as their respective classical Poisson brackets (75). Hence, Eq. (79) is only a formal one which must be worked out $[28,29,30]$.

Let us now see what is the Bohm-de Broglie interpretation of the solutions of Eqs. (76) and (77) in the metric and field representation. First we write the wave functional in polar form $\Psi=A \exp (i S / \hbar)$, where $A$ and $S$ are functionals of $h_{i j}$ and $\phi$. Substituting it in Eq. (78), we get two equations saying that $A$ and $S$ are invariant under general space coordinate transformations:

$$
\begin{aligned}
& -2 h_{l i} D_{j} \frac{\delta S\left(h_{i j}, \phi\right)}{\delta h_{l j}}+\frac{\delta S\left(h_{i j}, \phi\right)}{\delta \phi} \partial_{i} \phi=0, \\
& -2 h_{l i} D_{j} \frac{\delta A\left(h_{i j}, \phi\right)}{\delta h_{l j}}+\frac{\delta A\left(h_{i j}, \phi\right)}{\delta \phi} \partial_{i} \phi=0 .
\end{aligned}
$$

The two equations we obtain for $A$ and $S$ when we substitute $\Psi=A \exp (i S / \hbar)$ into Eq. (77) will of course depend on the factor ordering we choose. However, in any case, one of the equations will have the form

$$
\kappa G_{i j k l} \frac{\delta S}{\delta h_{i j}} \frac{\delta S}{\delta h_{k l}}+\frac{1}{2} h^{-1 / 2}\left(\frac{\delta S}{\delta \phi}\right)^{2}+V+Q=0,
$$

where $V$ is the classical potential given in Eq. (80). Contrary to the other terms in Eq. (83), which are already well defined, the precise form of $Q$ depends on the regularization and factor ordering which are prescribed for the Wheeler-DeWitt equation. In the unregulated form given in Eq. (79), $Q$ is

$$
Q=-\hbar^{2} \frac{1}{A}\left(\kappa G_{i j k l} \frac{\delta^{2} A}{\delta h_{i j} \delta h_{k l}}+\frac{h^{-1 / 2}}{2} \frac{\delta^{2} A}{\delta \phi^{2}}\right) .
$$

Also, the other equation besides (83) in this case is

$$
\kappa G_{i j k l} \frac{\delta}{\delta h_{i j}}\left(A^{2} \frac{\delta S}{\delta h_{k l}}\right)+\frac{1}{2} h^{-1 / 2} \frac{\delta}{\delta \phi}\left(A^{2} \frac{\delta S}{\delta \phi}\right)=0 .
$$

Let us now implement the Bohm-de Broglie interpretation for canonical quantum gravity. First of all we note that Eqs. (81) and (83), which are always valid irrespective of any factor ordering of the Wheeler-DeWitt equation, are like the Hamilton-Jacobi equations for GR, supplemented by an extra term $Q$ in the case of Eq. (83), which we will call the quantum potential. By analogy with the cases of non-relativistic particle and 
quantum field theory in flat spacetime, we will postulate that the 3-metric of spacelike hypersurfaces, the scalar field, and their canonical momenta always exist, independent on any observation, and that the evolution of the 3-metric and scalar field can be obtained from the guidance relations

$$
\begin{aligned}
\Pi^{i j} & =\frac{\delta S\left(h_{a b}, \phi\right)}{\delta h_{i j}}, \\
\Pi_{\phi} & =\frac{\delta S\left(h_{i j}, \phi\right)}{\delta \phi},
\end{aligned}
$$

with $\Pi^{i j}$ and $\Pi_{\phi}$ given by Eqs. (63) and (65), respectively. Like before, these are first order differential equations which can be integrated to yield the 3-metric and scalar field for all values of the $t$ parameter. These solutions depend on the initial values of the 3 -metric and scalar field at some initial hypersurface. The evolution of these fields will of course be different from the classical one due to the presence of the quantum potential term $Q$ in Eq. (83). The classical limit is once more conceptually very simple: it is given by the limit where the quantum potential $Q$ becomes negligible with respect to the classical energy. The only difference from the previous cases of the non-relativistic particle and quantum field theory in flat spacetime is the fact that the equivalent of Eqs. (3) and (7) for canonical quantum gravity, which in the naive ordering is Eq. (85), cannot be interpreted as a continuity equation for a probability density $A^{2}$ because of the hyperbolic nature of the DeWitt metric $G_{i j k l}$. However, even without a notion of probability, which in this case would mean the probability density distribution for initial values of the 3 -metric and scalar field in an initial hypersurface, we can extract a lot of information from Eq. (83) whatever is the quantum potential $Q$, as will see now. After we get these results, we will return to this probability issue in the last section.

First we note that, whatever is the form of the quantum potential $Q$, it must be a scalar density of weight one. This comes from the Hamilton-Jacobi equation (83). From this equation we can express $Q$ as

$$
Q=-\kappa G_{i j k l} \frac{\delta S}{\delta h_{i j}} \frac{\delta S}{\delta h_{k l}}-\frac{1}{2} h^{-1 / 2}\left(\frac{\delta S}{\delta \phi}\right)^{2}-V .
$$

As $S$ is an invariant (see Eq. (81)), then $\delta S / \delta h_{i j}$ and $\delta S / \delta \phi$ must be a second rank tensor density and a scalar density, both of weight one, respectively. When their products are contracted with $G_{i j k l}$ and multiplied by $h^{-1 / 2}$, respectively, they form a scalar density of weight one. As $V$ is also a scalar density of weight one, then $Q$ must also be. Furthermore, $Q$ must depend only on $h_{i j}$ and $\phi$ because it comes from the wave functional which depends only on these variables. Of course it can be non-local (we show an example in the appendix), i.e., depending on integrals of the fields over the whole space, but it cannot depend on the momenta.
Now we will investigate the following important problem. From the guidance relations (86) and (87), which will be written in the form

$$
\Phi^{i j} \equiv \Pi^{i j}-\frac{\delta S\left(h_{a b}, \phi\right)}{\delta h_{i j}} \approx 0
$$

and

$$
\Phi_{\phi} \equiv \Pi_{\phi}-\frac{\delta S\left(h_{i j}, \phi\right)}{\delta \phi} \approx 0
$$

we obtain the following first order partial differential equations:

$$
\dot{h}_{i j}=2 N G_{i j k l} \frac{\delta S}{\delta h_{k l}}+D_{i} N_{j}+D_{j} N_{i}
$$

and

$$
\dot{\phi}=N h^{-1 / 2} \frac{\delta S}{\delta \phi}+N^{i} \partial_{i} \phi
$$

The question is, given some initial 3-metric and scalar field, what kind of structure do we obtain when we integrate this equations in the parameter $t$ ? Does this structure form a 4-dimensional geometry with a scalar field for any choice of the lapse and shift functions? Note that if the functional $S$ were a solution of the classical Hamilton-Jacobi equation, which does not contain the quantum potential term, then the answer would be in the affirmative because we would be in the scope of GR. But $S$ is a solution of the modified Hamilton-Jacobi equation (83), and we cannot guarantee that this will continue to be true. We may obtain a complete different structure due to the quantum effects driven by the quantum potential term in Eq. (83). To answer this question we will move from this Hamilton-Jacobi picture of quantum geometrodynamics to a hamiltonian picture. This is because many strong results concerning geometrodynamics were obtained in this later picture [25,37]. We will construct a hamiltonian formalism which is consistent with the guidance relations (86) and (87). It yields the bohmian trajectories (91) and (92) if the guidance relations are satisfied initially. Once we have this hamiltonian, we can use well known results in the literature to obtain strong results about the Bohmde Broglie view of quantum geometrodynamics.

Examining Eqs. (81) and (83), we can easily show [20] that the hamiltonian which generates the bohmian trajectories, once the guidance relations (86) and (87) are satisfied initially, is given by:

$$
H_{Q}=\int d^{3} x\left[N(\mathcal{H}+Q)+N^{i} \mathcal{H}_{i}\right]
$$

where we define

$$
\mathcal{H}_{Q} \equiv \mathcal{H}+Q \text {. }
$$

The quantities $\mathcal{H}$ and $\mathcal{H}_{i}$ are the usual GR superhamiltonian and super-momentum constraints given by Eqs. (61) and (62). In fact, the guidance relations (86) and $(87)$ are consistent with the constraints $\mathcal{H}_{Q} \approx 0$ 
and $\mathcal{H}_{i} \approx 0$ because $S$ satisfies (81) and (83). Furthermore, they are conserved by the hamiltonian evolution given by (93) [20].

We now have a hamiltonian, $H_{Q}$, which generates the bohmian trajectories once the guidance relations (86) and (87) are imposed initially. In the following, we can investigate if the the evolution of the fields driven by $H_{Q}$ forms a four-geometry like in classical geometrodynamics. First we recall a result obtained by Claudio Teitelboim [37]. In this paper, he shows that if the 3-geometries and field configurations defined on hypersurfaces are evolved by some hamiltonian with the form

$$
\bar{H}=\int d^{3} x\left(N \overline{\mathcal{H}}+N^{i} \overline{\mathcal{H}}_{i}\right)
$$

and if this evolution can be viewed as the "motion" of a 3-dimensional cut in a 4-dimensional spacetime (the 3 geometries can be embedded in a four-geometry), then the constraints $\overline{\mathcal{H}} \approx 0$ and $\overline{\mathcal{H}}_{i} \approx 0$ must satisfy the following algebra

$$
\begin{aligned}
\left\{\overline{\mathcal{H}}(x), \overline{\mathcal{H}}\left(x^{\prime}\right)\right\} & =-\epsilon\left[\overline{\mathcal{H}}^{i}(x) \partial_{i} \delta^{3}\left(x^{\prime}, x\right)\right]-\overline{\mathcal{H}}^{i}\left(x^{\prime}\right) \partial_{i} \delta^{3}\left(x, x^{\prime}\right) \\
\left\{\overline{\mathcal{H}}_{i}(x), \overline{\mathcal{H}}\left(x^{\prime}\right)\right\} & =\overline{\mathcal{H}}(x) \partial_{i} \delta^{3}\left(x, x^{\prime}\right) \\
\left\{\overline{\mathcal{H}}_{i}(x), \overline{\mathcal{H}}_{j}\left(x^{\prime}\right)\right\} & =\overline{\mathcal{H}}_{i}(x) \partial_{j} \delta^{3}\left(x, x^{\prime}\right)-\overline{\mathcal{H}}_{j}\left(x^{\prime}\right) \partial_{i} \delta^{3}\left(x, x^{\prime}\right)
\end{aligned}
$$

The constant $\epsilon$ in (96) can be \pm 1 depending if the fourgeometry in which the 3 -geometries are embedded is euclidean $(\epsilon=1)$ or hyperbolic $(\epsilon=-1)$. These are the conditions for the existence of spacetime.

The above algebra is the same as the algebra (75) of GR if we choose $\epsilon=-1$. But the hamiltonian (93) is different from the hamiltonian of GR only by the presence of the quantum potential term $Q$ in $\mathcal{H}_{Q}$. The Poisson bracket $\left\{\mathcal{H}_{i}(x), \mathcal{H}_{j}\left(x^{\prime}\right)\right\}$ satisfies Eq. (98) because the $\mathcal{H}_{i}$ of $H_{Q}$ defined in Eq. (93) is the same as in GR. Also $\left\{\mathcal{H}_{i}(x), \mathcal{H}_{Q}\left(x^{\prime}\right)\right\}$ satisfies Eq. (97) because $\mathcal{H}_{i}$ is the generator of spatial coordinate transformations, and as $\mathcal{H}_{Q}$ is a scalar density of weight one (remember that $Q$ must be a scalar density of weight one), then it must satisfies this Poisson bracket relation with $\mathcal{H}_{i}$. What remains to be verified is if the Poisson bracket $\left\{\mathcal{H}_{Q}(x), \mathcal{H}_{Q}\left(x^{\prime}\right)\right\}$ closes as in Eq. (96). We now recall the result of Ref. [25]. There it is shown that a general super-hamiltonian $\overline{\mathcal{H}}$ which satisfies Eq. (96), is a scalar density of weight one, whose geometrical degrees of freedom are given only by the three-metric $h_{i j}$ and its canonical momentum, and contains only even powers and no non-local term in the momenta (together with the other requirements, these last two conditions are also satisfied by $\mathcal{H}_{Q}$ because it is quadratic in the momenta and the quantum potential does not contain any non-local term on the momenta), then $\overline{\mathcal{H}}$ must have the following form:

$$
\overline{\mathcal{H}}=\kappa G_{i j k l} \Pi^{i j} \Pi^{k l}+\frac{1}{2} h^{-1 / 2} \pi_{\phi}^{2}+V_{G},
$$

where

$V_{G} \equiv-\epsilon h^{1 / 2}\left[-\kappa^{-1}\left(R^{(3)}-2 \bar{\Lambda}\right)+\frac{1}{2} h^{i j} \partial_{i} \phi \partial_{j} \phi+\bar{U}(\phi)\right]$

With this result we can now establish two possible scenarios for the Bohm-de Broglie quantum geometrodynamics, depending on the form of the quantum potential:

\section{IV.1. Quantum geometrodynamics evo- lution is consistent and forms a non de- generate four-geometry}

In this case, the Poisson bracket $\left\{\mathcal{H}_{Q}(x), \mathcal{H}_{Q}\left(x^{\prime}\right)\right\}$ must satisfy Eq. (96). Then $Q$ must be such that $V+Q=V_{G}$ with $V$ given by (80) yielding:

$$
Q=-h^{1 / 2}\left[(\epsilon+1)\left(-\kappa^{-1} R^{(3)}+\frac{1}{2} h^{i j} \partial_{i} \phi \partial_{j} \phi\right)+\frac{2}{\kappa}(\epsilon \bar{\Lambda}+\Lambda)+\epsilon \bar{U}(\phi)+U(\phi)\right]
$$


Then we have two possibilities:

\section{IV.1.1. The spacetime is hyperbolic $(\epsilon=-1)$}

In this case $Q$ is

$$
Q=-h^{1 / 2}\left[\frac{2}{\kappa}(-\bar{\Lambda}+\Lambda)-\bar{U}(\phi)+U(\phi)\right] .
$$

Hence $Q$ is like a classical potential. Its effect is to renormalize the cosmological constant and the classical scalar field potential, nothing more. The quantum geometrodynamics is indistinguishable from the classical one. It is not necessary to require the classical limit $Q=0$ because $V_{G}=V+Q$ already may describe the classical universe we live in.

\section{IV.1.2. The spacetime is euclidean $(\epsilon=1)$}

In this case $Q$ is

$$
Q=-h^{1 / 2}\left[2\left(-\kappa^{-1} R^{(3)}+\frac{1}{2} h^{i j} \partial_{i} \phi \partial_{j} \phi\right)+\frac{2}{\kappa}(\bar{\Lambda}+\Lambda)+\bar{U}(\phi)+U(\phi)\right] .
$$

Now $Q$ not only renormalize the cosmological constant and the classical scalar field potential but also change the signature of spacetime. The total potential $V_{G}=V+Q$ may describe some era of the early universe when it had euclidean signature, but not the present era, when it is hyperbolic. The transition between these two phases must happen in a hypersurface where $Q=0$, which is the classical limit.

We can conclude from these considerations that if a quantum spacetime exists with different features from the classical observed one, then it must be euclidean. In other words, the sole relevant quantum effect which maintains the non-degenerate nature of the four-geometry of spacetime is its change of signature to a euclidean one. The other quantum effects are either irrelevant or break completely the spacetime structure. This result points in the direction of Ref. [38].

\section{IV.2. Quantum geometrodynamics evo- lution is consistent but does not form a non degenerate four-geometry}

In this case, the Poisson bracket $\left\{\mathcal{H}_{Q}(x), \mathcal{H}_{Q}\left(x^{\prime}\right)\right\}$ does not satisfy Eq. (96) but is weakly zero in some other way. Some examples are given in Ref. [40]. They are real solutions of the Wheeler-DeWitt equation, where $Q=-V$, and non-local quantum potentials. It is very important to use the guidance relations to close the algebra in these cases. It means that the hamiltonian evolution with the quantum potential is consistent only when restricted to the bohmian trajectories. For other trajectories, it is inconsistent. Concluding, when restricted to the bohmian trajectories, an algebra which does not close in general may close, as shown in the above example. This is an important remark on the Bohm-de Broglie interpretation of canonical quantum cosmology, which sometimes is not noticed.

In the examples above, we have explicitly obtained the "structure constants" of the algebra that characterizes the "pre-four-geometry" generated by $H_{Q}$ i.e., the foam-like structure pointed long time ago in early works of J. A. Wheeler [35, 42].

\section{Conclusion and Discussions}

The Bohm-de Broglie interpretation of canonical quantum cosmology yields a quantum geometrodynamical picture where the bohmian quantum evolution of threegeometries may form, depending on the wave functional, a consistent non degenerate four geometry which must be euclidean (but only for a very special local form of the quantum potential), and a consistent but degenerate four-geometry indicating the presence of special vector fields and the breaking of the spacetime structure as a single entity (in a wider class of possibilities). Hence, in general, and always when the quantum potential is non-local, spacetime is broken. The threegeometries evolved under the influence of a quantum potential do not in general stick together to form a non degenerate four-geometry, a single spacetime with the causal structure of relativity. This is not surprising, as it was anticipated long ago [42]. Among the consistent bohmian evolutions, the more general structures that are formed are degenerate four-geometries with alternative causal structures. We obtained these results taking a minimally coupled scalar field as the matter source of gravitation, but it can be generalized to any matter source with non-derivative couplings with the metric, like Yang-Mills fields.

As shown in the previous section, a non degenerate four-geometry can be attained only if the quantum potential have the specific form (101). In this case, the sole relevant quantum effect will be a change of signature of spacetime, something pointing towards Hawking's ideas.

In the case of consistent quantum geometrodynam- 
ical evolution but with degenerate four-geometry, we have shown that any real solution of the WheelerDeWitt equation yields a structure which is the idealization of the strong gravity limit of GR. This type of geometry, which is degenerate, has already been studied [41]. Due to the generality of this picture (it is valid for any real solution of the Wheeler-DeWitt equation, which is a real equation), it deserves further attention. It may well be that these degenerate four-metrics were the correct quantum geometrodynamical description of the young universe. It would be also interesting to investigate if these structures have a classical limit yielding the usual four-geometry of classical cosmology.

For non-local quantum potentials, we have shown that apparently inconsistent quantum evolutions are in fact consistent if restricted to the bohmian trajectories satisfying the guidance relations (86) and (87). This is a point which is sometimes not taken into account.

If we want to be strict and impose that quantum geometrodynamics does not break spacetime, then we will have stringent boundary conditions. As said above, a non degenerate four-geometry can be obtained only if the quantum potential have the form (101). This is a severe restriction on the solutions of the Wheeler-DeWitt equation.

These restrictions on the form of the quantum potential do not occur in minisuperspace models [19] because there the hypersurfaces are restricted to be homogeneous. The only freedom we have is in the time parametrization of the homogeneous hypersurfaces which foliate spacetime. There is a single constraint, which of course always commute with itself, irrespective of the quantum potential. The theorem proven in Ref. [25], which was essential in all the reasoning of the last section, cannot be used here because minisuperspace models do not satisfy one of their hypotheses. In section 3 we studied quantum effects in such minisuperspace models and we showed that they can avoid singularities, isotropize the Universe, and create inflationary epochs. It should be very interesting to investigate if these quantum phases of the Universe may have left some traces which could be detected now, as in the anisotropies of the cosmic microwave background radiation.

As we have seen, in the Bohm-de Broglie approach we can investigate further what kind of structure is formed in quantum geometrodynamics by using the Poisson bracket relation (96), and the guidance relations (91) and (92). By assuming the existence of 3geometries, field configurations, and their momenta, independently on any observations, the Bohm-de Broglie interpretation allows us to use classical tools, like the hamiltonian formalism, to understand the structure of quantum geometry. If this information is useful, we do not know. Already in the two-slit experiment in nonrelativistic quantum mechanics, the Bohm-de Broglie interpretation allows us to say from which slit the par- ticle has passed through: if it arrive at the upper half of the screen it must have come from the upper slit, and vice-versa. Such information we do not have in the many-worlds interpretation. However, this information is useless: we can neither check it nor use it in other experiments. In canonical quantum cosmology the situation may be the same. The Bohm-de Broglie interpretation yields a lot of information about quantum geometrodynamics which we cannot obtain from the many-worlds interpretation, but this information may be useless. However, we cannot answer this question precisely if we do not investigate further, and the tools are at our disposal.

We would like to remark that all these results were obtained without assuming any particular factor ordering and regularization of the Wheeler-DeWitt equation. Also, we did not use any probabilistic interpretation of the solutions of the Wheeler-DeWitt equation. Hence, it is a quite general result. However, we would like to make some comments about the probability issue in quantum cosmology. The Wheeler-DeWitt equation when applied to a closed universe does not yield a probabilistic interpretation for their solutions because of its hyperbolic nature. However, it has been suggested many times $[21,43,44,45,46]$ that at the semiclassical level we can construct a probability measure with the solutions of the Wheeler-DeWitt equation. Hence, for interpretations where probabilities are essential, the problem of finding a Hilbert space for the solutions of the Wheeler-DeWitt equation becomes crucial if someone wants to get some information above the semiclassical level. Of course, probabilities are also useful in the Bohm-de Broglie interpretation. When we integrate the guidance relations (91) and (92), the initial conditions are arbitrary, and it should be nice to have some probability distribution on them. However, as we have seen along this paper, we can extract a lot of information from the full quantum gravity level using the Bohm-de Broglie interpretation, without appealing to any probabilistic notion.

It would also be important to investigate the Bohmde Broglie interpretation for other quantum gravitational systems, like black holes. Attempts in this direction have been made, but within spherical symmetry in empty space [47], where we have only a finite number of degrees of freedom. It should be interesting to investigate more general models.

The conclusions of this paper are of course limited by many strong assumptions we have tacitly made, as supposing that a continuous three-geometry exists at the quantum level (quantum effects could also destroy it), or the validity of quantization of standard GR, forgetting other developments like string theory. However, even if this approach is not the appropriate one, it is nice to see how far we can go with the Bohm-de Broglie interpretation, even in such incomplete stage of canonical quantum gravity. It seems that the Bohm- 
de Broglie interpretation may at least be regarded as a nice "gauge" [48] to be used in quantum cosmology, as, probably, it will prove harder, or even impossible, to reach the detailed conclusions of this paper using other interpretations. Furthermore, if the finer view of the Bohm-de Broglie interpretation of quantum cosmology can yield useful information in the form of observational effects, then we will have means to decide between interpretations, something that will be very important not only for quantum cosmology, but for quantum theory itself.

\section{Acknowledgments}

We would like to thank CNPq of Brazil for financial support.

\section{References}

[1] N. Bohr, Atomic Physics and Human Knowledge (Science Editions, New York, 1961); N. Bohr; Phys. Rev. 48, 696 (1935).

[2] W. Heisenberg, The Physical Principles of the Quantum Theory (Dover, New York, 1949).

[3] J. von Neumann, Mathematical Foundations of Quantum Mechanics (Princeton University Press, Princeton, 1955).

[4] R. Omnès, The Interpretation of Quantum Mechanics (Princeton University Press, Princeton, 1994).

[5] H. D. Zeh, Found. Phys. 1, 69 (1970); E. Joos and H. D. Zeh, Z. Phys. B 59, 223 (1985); W. H. Zurek, Phys. Rev. D 26, 1862 (1982); W. H. Zurek, Phys. Today 44, 36 (1991)

[6] C. Kiefer, Class. Quantum Grav. 18, 379 (1991); D. Giulini, E. Joos, C. Kiefer, J. Kupsch, I. O. Stamatescu and H. D. Zeh, Decoherence and the Appearance of a Classical World in Quantum Theory (Springer-Verlag, Berlin, 1996).

[7] V.F Mukhanov, in Physical Origins of Time Asymmetry, ed by J. J. Halliwell, J. Pérez-Mercader and W. H. Zurek (Cambridge University Press, 1994).

[8] H. D. Zeh, in Decoherence and the Appearance of a Classical World in Quantum Theory (Springer-Verlag, Berlin, 1996).

[9] M. Gell-Mann and J. B. Hartle, in Complexity, Entropy and the Physics of Information, ed. by W. H. Zurek (Addison Wesley, 1990).

[10] J. P. Paz and W. H. Zurek, Phys. Rev. D 48, 2728 (1993).

[11] G.C. Ghirardi, A. Rimini and T. Weber, Phys. Rev. D 34470 (1986); G.C. Ghirardi , P. Pearle and A. Rimini, Phys. Rev. A 42, 78 (1990).

[12] R. Penrose, in Quantum Implications: Essays in Honour of David Bohm, ed. by B. J. Hiley and F. David Peat (Routledge, London, 1987).
[13] The Many-Worlds Interpretation of Quantum Mechanics, ed. by B. S. DeWitt and N. Graham (Princeton University Press, Princeton, 1973).

[14] D. Bohm, Phys. Rev. 85, 166 (1952); D. Bohm, B. J. Hiley and P. N. Kaloyerou, Phys. Rep. 144, 349 (1987).

[15] P. R. Holland, The Quantum Theory of Motion: An Account of the de Broglie-Bohm Causal Interpretation of Quantum Mechanics (Cambridge University Press, Cambridge, 1993).

[16] J. C. Vink, Nucl. Phys. B 369, 707 (1992).

[17] Y. V. Shtanov, Phys. Rev. D 54, 2564 (1996).

[18] A. Valentini, Phys. Lett. A 158, 1, (1991).

[19] J. A. de Barros and N. Pinto-Neto, Int. J. of Mod. Phys. D 7, 201 (1998).

[20] N. Pinto-Neto and E. S. Santini, Phys. Rev. D 59, 123517, (1999).

[21] J. Kowalski-Glikman and J. C. Vink, Class. Quantum Grav. 7, 901 (1990).

[22] E. J. Squires, Phys. Lett. A 162, 35 (1992).

[23] J. A. de Barros, N. Pinto-Neto and M. A. Sagioro-Leal, Phys. Lett. A 241, 229 (1998).

[24] R. Colistete Jr., J. C. Fabris and N. Pinto-Neto, Phys. Rev. D 57, 4707 (1998).

[25] S. A. Hojman, K. Kuchař and C. Teitelboim, Ann. Phys. 96, 88 (1976).

[26] E. Cartan, Annales Scientifiques de l'Ecole Normale Supérieure 40, 325 (1923) and 41, 1 (1924).

[27] N. C. Tsamis and R. P. Woodward, Phys. Rev. D 36, 3641 (1987).

[28] K. Maeda and M. Sakamoto, Phys. Rev. D 54, 1500 (1996)

[29] T. Horiguchi, K. Maeda and M. Sakamoto, Phys. Lett. B 344, 105 (1995).

[30] J. Kowalski-Glikman and K. A. Meissner, Phys. Lett. B 376, 48 (1996).

[31] J. M. Lévy Leblond, Ann. Inst. Henri Poincarè 3, 1 (1965).

[32] D. Bohm and J. P. Vigier, Phys. Rev. 96, 208 (1954).

[33] A. Valentini, Phys. Lett. A 156, 5 (1991).

[34] P. N. Kaloyerou, Phys. Rep. 244, 287 (1994).

[35] J.A. Wheeler, in Battelle Rencontres: 1967 Lectures in Mathematical Physics, ed. by B. DeWitt and J.A.Wheeler (Benjamin New York, 1968).

[36] B. S. DeWitt, Phys. Rev. 160, 1113 (1967).

[37] C. Teitelboim, Ann. Phys. 80, 542 (1973).

[38] Euclidean Quantum Gravity, ed. by G. W. Gibbons and S. W. Hawking (World Scientific, London, 1993).

[39] C. Teitelboim, Phys. Rev. D 25, 3159 (1982).

[40] N. Pinto- Neto and E. Sergio Santini, 'Geometrodinâmica quântica na interpretação de Bohm- de Broglie: o espaço- tempo quântico deve ser euclideano?', this volume.

[41] G. Dautcourt, report gr-gc/9801093. 
[42] J. A. Wheeler, Ann. Phys. 2, 604 (1957); J. A. Wheeler, in Relativity, Groups and Topology, ed. by B. DeWitt and C. DeWitt (Gordon and Breach, New York, 1964); G.M Patton and J.A Wheeler, in Quantum Gravity. An Oxford Symposium, ed. by C.J. Isham, R.Penrose and D. Sciama (Clarendon Press, Oxford, 1975).

[43] T. Banks, Nucl. Phys. B 249, 332 (1985).

[44] T. P. Singh and T. Padmanabhan, Ann. Phys. 196, 296 (1989).

[45] D. Giulini and C. Kiefer, Class. Quantum Grav. 12, 403 (1995).
[46] J.J. Halliwell, in Quantum Cosmology and Baby Universes, ed. by S. Coleman, J.B. Hartle, T. Piran and S. Weinberg (World Scientific, Singapore, 1991).

[47] M. Kenmoku, H. Kubotani, E. Takasugi and Y. Yamazaki, report gr-qc 9810039.

[48] We thank this image to Brandon Carter.

[49] K. Kuchař , Phys. Rev. D 50, 3961 (1994).

[50] J. Louko and S. N. Winters-Hilt, Phys. Rev. D 54, 2647 (1996).

[51] T. Brotz and C. Kiefer, Phys. Rev. D 55, 2186 (1997). 